\title{
On the Use of a Multivariable Frequency Response Estimation Method for Closed Loop Identification
}

\author{
Erik Wernholt, Svante Gunnarsson \\ Division of Automatic Control \\ Department of Electrical Engineering \\ Linköpings universitet, SE-581 83 Linköping, Sweden \\ WWW: http://www. control.isy.liu.se \\ E-mail: erikw@isy.liu.se, svante@isy.liu.se
}

29th March 2004

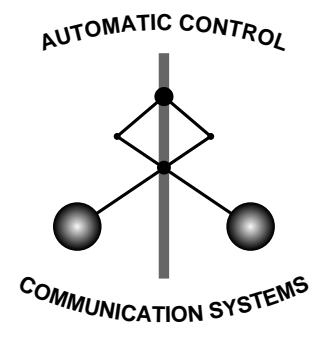

LINKÖPING

\author{
Report no.: LiTH-ISY-R-2600
}

Submitted to CDC'04

Technical reports from the Control \& Communication group in Linköping are available at http://www.control.isy.liu.se/publications. 


\begin{abstract}
A method for estimating the Multivariable Frequency Response Function using closed loop data is studied. An approximate expression for the estimation error is derived, and using this expression some properties of the estimation error can be explained. Of particular interest is how the model quality is affected by the properties of the disturbances, the choice of excitation signal in the different input channels, the feedback and the properties of the system itself. The expression is illustrated by simulation data from an industrial robot.
\end{abstract}

Keywords: closed loop identification, multivariable system, frequency response, periodic disturbances, excitation signals, robotics 


\title{
On the Use of a Multivariable Frequency Response Estimation Method for Closed Loop Identification
}

\author{
Erik Wernholt and Svante Gunnarsson \\ Department of Electrical Engineering \\ Linköpings universitet \\ SE-58183 Linköping, Sweden \\ \{erikw, svante\}@isy.liu.se
}

\begin{abstract}
A method for estimating the Multivariable Frequency Response Function using closed loop data is studied. An approximate expression for the estimation error is derived, and using this expression some properties of the estimation error can be explained. Of particular interest is how the model quality is affected by the properties of the disturbances, the choice of excitation signal in the different input channels, the feedback and the properties of the system itself. The expression is illustrated by simulation data from an industrial robot.
\end{abstract}

\section{INTRODUCTION}

Accurate dynamical models are needed in many applications, e.g. for analysis, simulation, controller design, and diagnosis. Two main routes to obtain these models are modeling and system identification, i.e., using previous experience (based on previous empirical work) and experimental data, respectively. System identification is a vast research area, and several text books are available. See e.g. [1] and [2].

It is sometimes necessary to perform identification experiments under output feedback, i.e., in closed loop. The reason may, e.g., be an unstable plant, or that is has to be controlled for production, economic, or safety reasons. Most (non-parametric) estimation methods of the plant frequency response function usually only consider systems with scalar input and output (see, e.g., the ETFE method in [1]).

Here we will consider an estimation method, described in [3], for the multivariable frequency response function. In particular, the method is evaluated with respect to the properties of the disturbances and the excitation signals. An approximate expression for the estimation error is derived, and using this expression some properties of the estimation error can be explained.

For a numerical example, we will use simulations of an industrial robot. The robot application is interesting since it gives many challenging problems for system identification methods, such as a multivariable non-linear system, oscillatory behavior, closed loop identification (to stay centered around the working point), disturbances, and non-linearities such as e.g. static friction. An overview of identification in robotics can be found in [4].

The numerical experiments are carried out using a linearization of a 20th order non-linear simulation model of a modern industrial robot. The reason for using a simulation model is that it makes it possible to study the different aspects separately. By, for example, keeping the character of the excitation signal fixed, and changing the properties of the disturbances, and, in addition, having the true model available, it is possible to get insight into the properties of the estimated model. The results from the identification experiments are evaluated using the approximate, but explicit, expression for the estimation error.

The paper is organized as follows. Section II presents the frequency domain identification method that will be used in the paper, and Section III deals with the problem of selecting the excitation signals for the data collection. An approximate error expression is derived in Section IV, which is illustrated by a numerical example in Section V. Finally, Section VI contains some conclusions.

\section{The IDENTIFICATION METHOD}

The identification method that will be used is described in [3] and it is an estimation method for a multivariable frequency response function (MFRF). Consider the setting in Fig. 1, where $F$ is the controller and $G$ is the plant to identify. The controller takes as input the difference between the reference signal $r$ and the measured plant output $y$, and $u$ is the commanded plant input. Due to disturbances $v_{u}$ and $v_{y}$, the plant input will be $u_{s}=u+v_{u}$ and the measured plant output $y=y_{s}+v_{y}$, i.e. the sum of the true output $y_{s}$ and the output disturbance $v_{y}$.

First, consider the disturbance free case $\left(v_{u}=v_{y}=0\right)$. Let $G(z)$ be the discrete time multivariable plant with $m$ inputs and $p$ outputs. The measured input and output signals, $u(t) \in \mathbb{R}^{m}$ and $y(t) \in \mathbb{R}^{p}$, are sampled at time instants $t_{n}=n T_{s}, n=1,2, \ldots, N$, with $T_{s}$ the sample time and

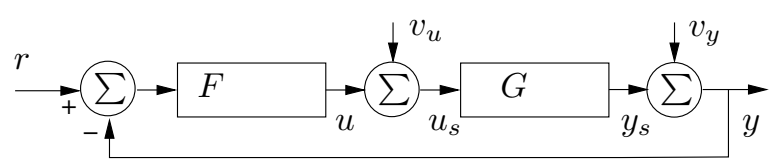

Fig. 1. Block diagram. 
$T_{0}=N T_{s}$ the signal period. Let

$$
\begin{aligned}
& U_{N}\left(\omega_{k}\right)=\frac{1}{\sqrt{N}} \sum_{n=1}^{N} u\left(n T_{s}\right) e^{i \omega_{k} n T_{s}}, \\
& Y_{N}\left(\omega_{k}\right)=\frac{1}{\sqrt{N}} \sum_{n=1}^{N} y\left(n T_{s}\right) e^{i \omega_{k} n T_{s}},
\end{aligned}
$$

be the DFT of the sampled signals, where

$$
\omega_{k}=k \frac{2 \pi}{N T_{s}}, \quad k=1,2, \ldots, N .
$$

If the sampled signals are periodic, the following linear mapping will hold exactly

$$
Y_{N}\left(\omega_{k}\right)=G\left(e^{i \omega_{k} T_{s}}\right) U_{N}\left(\omega_{k}\right),
$$

where $G\left(e^{i \omega_{k} T_{s}}\right) \in \mathbb{C}^{p \times m}$ is the MFRF. We will in the sequel use $G\left(\omega_{k}\right)$ as a short notation for $G\left(e^{i \omega_{k} T_{s}}\right)$.

Note that if the sampled signals are not periodic, the DFT will introduce leakage errors due to the limited time window and (1) will no longer hold exactly (see Section 2.2.2. in [2] for details). This is the main reason why only periodic excitation is considered.

To be able to extract $G\left(\omega_{k}\right)$ from data, at least $m$ different (independent) experiments are needed. The data vectors from the different experiments can then be collected into matrices (bold face in the sequel) where each column corresponds to one experiment. The relation between the input and output can then be written as

$$
\mathbf{Y}_{N}\left(\omega_{k}\right)=G\left(\omega_{k}\right) \mathbf{U}_{N}\left(\omega_{k}\right),
$$

where $\mathbf{U}_{N}\left(\omega_{k}\right) \in \mathbb{C}^{m \times m}$ and $\mathbf{Y}_{N}\left(\omega_{k}\right) \in \mathbb{C}^{p \times m}$. If $\mathbf{U}_{N}\left(\omega_{k}\right)$ has full rank, an estimate of $G\left(\omega_{k}\right)$ can be formed as

$$
\hat{G}_{N}\left(\omega_{k}\right)=\mathbf{Y}_{N}\left(\omega_{k}\right) \mathbf{U}_{N}^{-1}\left(\omega_{k}\right) .
$$

If more than $m$ experiments are carried out, $\mathbf{U}_{N}\left(\omega_{k}\right)^{-1}$ can be replaced by the pseudo-inverse.

Usually, input/output measurements are corrupted by disturbances. To reduce the variance of the estimate, the periodic excitation can be repeated and use averaged versions of $\mathbf{Y}_{N}\left(\omega_{k}\right)$ and $\mathbf{U}_{N}^{-1}\left(\omega_{k}\right)$ (see [3] for details).

In order to evaluate the quality of the estimated models, some quality measures are needed. In [5] there are bias expressions for the FRF estimate in the SISO case in the presence of correlated input/output errors. Here we will mainly look at the relative error of the estimate, defined as

$$
\Delta G_{N}\left(\omega_{k}\right)=\frac{\hat{G}_{N}\left(\omega_{k}\right)-G\left(\omega_{k}\right)}{G\left(\omega_{k}\right)} .
$$

For MIMO systems, which is the case here, the relative error is calculated element-wise. For an easier comparison, the absolute value of the relative error, $\left|\Delta G_{N}\left(\omega_{k}\right)\right|$, is averaged over the excited frequency interval, $\Omega$, as in

$$
\overline{\left|\Delta G_{N}\right|}=\frac{1}{N_{\Omega}} \sum_{\omega_{k} \in \Omega}\left|\Delta G_{N}\left(\omega_{k}\right)\right|,
$$

where $N_{\Omega}$ is the number of excited frequencies. The subscript $N$ will from now on be omitted for easier notation.

\section{Excitation Signals}

The selection of excitation signals is an important step in the design of good experiments. For a detailed treatment of different excitation signals, see e.g. [1] and [2]. What is an optimal excitation signal depends on the goal of the experiment. Here we will focus on the non-parametric approach and the energy should then be distributed to achieve a predefined accuracy in the frequency band of interest. The excitation signal will have a specified maximum peak value.

As was pointed out in Section II, we need (at least) the same number of experiments as the number of inputs. Consider therefore the matrix $\mathbf{r}(t) \in \mathbb{C}^{p \times m}$ of reference signals, where column $i$ corresponds to the reference signal applied in experiment $i$. How should $\mathbf{r}(t)$ be selected? In addition to the reference signal, the controller could be considered as a tuning parameter for the identification experiment. That is not considered in this article.

In Section II we mentioned that one should use periodic excitation for the MFRF estimation method to work as well as possible. Using a periodic reference signal will give periodic signals in steady state. However, for a poorly damped system like a robot, transients will make the required waiting time, $T_{w}$, long. With a suitable controller, the damping of the closed loop system can be increased, which reduces the required waiting time. To reduce the transient effects, we will here wait a couple of signal periods until no difference can be seen in the estimates from one signal period to the next. For estimations of the required waiting time, see e.g. [6] where approximate expressions are derived, based on the damping of the dominant poles.

We will here restrict our selection of $\mathbf{r}(t)$ to

$$
\mathbf{r}(t)=\operatorname{Tr}_{0}(t),
$$

where $r_{0}(t)$ is a scalar signal, and $T$ is a permutation matrix.Two cases of $T$ will be used for comparison

$$
T_{1}=\left(\begin{array}{lll}
1 & 0 & 0 \\
0 & 1 & 0 \\
0 & 0 & 1
\end{array}\right), T_{2}=\left(\begin{array}{ccc}
1 & 1 & 1 \\
1 & -1 & 1 \\
1 & 1 & -1
\end{array}\right),
$$

where the second matrix maximizes $\operatorname{det} T$ (only the set ($1,0,1)$ is allowed for the elements) and is suggested in [3] for the open loop case.

For the scalar $r_{0}(t)$ in (6) we will use the periodic multisine signal

$$
r_{0}(t)=\sum_{k=1}^{F} A_{k} \sin \left(2 \pi f_{k} t+\phi_{k}\right),
$$

with amplitudes $A_{k}$, phases $\phi_{k}$, signal period $T_{0}$, and frequencies $f_{k}=l_{k} f_{0}=l_{k} / T_{0}$ with $l_{k} \in \mathbb{N}$. The phases $\phi_{k}$ are chosen to get a low crest factor (ratio between peak value and root mean square value, see [2] for details). Random initial phases are combined with an iterative optimization procedure described in [7], which is referred as the clipping algorithm in the literature. 


\section{ERROR ANALYSIS}

Consider once more the block diagram in Fig. 1 for the case $v_{y} \neq 0$ and/or $v_{u} \neq 0$. How is the relative error (4) affected by the disturbances? We will here mainly consider the DFT matrices (bold face) of the signals, where column $i$ corresponds to experiment $i$. Now let $\mathbf{V}\left(\omega_{k}\right)$ denote the sum of the contributions from both input and output disturbances, i.e.

$$
\mathbf{V}\left(\omega_{k}\right)=\mathbf{V}_{y}\left(\omega_{k}\right)+G\left(\omega_{k}\right) \mathbf{V}_{u}\left(\omega_{k}\right)
$$

As measurements, we will consider the control signal calculated by the controller, $u$, and the measured output signal, $y$, given by

$$
\begin{aligned}
& \mathbf{U}\left(\omega_{k}\right)=G_{u}\left(\omega_{k}\right)\left(\mathbf{R}\left(\omega_{k}\right)-\mathbf{V}\left(\omega_{k}\right)\right), \\
& \mathbf{Y}\left(\omega_{k}\right)=G_{c}\left(\omega_{k}\right) \mathbf{R}\left(\omega_{k}\right)+S\left(\omega_{k}\right) \mathbf{V}\left(\omega_{k}\right),
\end{aligned}
$$

respectively, where

$$
\begin{aligned}
S\left(\omega_{k}\right) & =\left(I+G\left(\omega_{k}\right) F\left(\omega_{k}\right)\right)^{-1}, \\
G_{u}\left(\omega_{k}\right) & =\left(I+F\left(\omega_{k}\right) G\left(\omega_{k}\right)\right)^{-1} F\left(\omega_{k}\right), \\
G_{c}\left(\omega_{k}\right) & =G\left(\omega_{k}\right) F\left(\omega_{k}\right) S\left(\omega_{k}\right) .
\end{aligned}
$$

Lemma 1: Consider the setting in Fig. 1 with the estimator $\hat{G}\left(\omega_{k}\right)$ given by (3). The estimation error (neglecting leakage effects in the DFT:s) is

$$
\tilde{G}\left(\omega_{k}\right)=\hat{G}\left(\omega_{k}\right)-G\left(\omega_{k}\right)=\mathbf{V}\left(\omega_{k}\right) \mathbf{U}^{-1}\left(\omega_{k}\right),
$$

with $\mathbf{V}\left(\omega_{k}\right)$ and $\mathbf{U}\left(\omega_{k}\right)$ given by (9) and (10).

Proof: From (3) one gets

$$
\begin{aligned}
\tilde{G}\left(\omega_{k}\right) & =\hat{G}\left(\omega_{k}\right)-G\left(\omega_{k}\right) \\
& =\underbrace{\left(\mathbf{Y}\left(\omega_{k}\right)-G\left(\omega_{k}\right) \mathbf{U}\left(\omega_{k}\right)\right)}_{\mathbf{V}} \mathbf{U}^{-1}\left(\omega_{k}\right),
\end{aligned}
$$

which then immediately gives the desired result.

Using (10) the expression for the error can be rewritten as

$$
\tilde{G}\left(\omega_{k}\right)=\mathbf{V}\left(\omega_{k}\right)\left(\left(\mathbf{R}\left(\omega_{k}\right)-\mathbf{V}\left(\omega_{k}\right)\right)^{-1} G_{u}^{-1}\left(\omega_{k}\right),\right.
$$

where

$$
G_{u}^{-1}\left(\omega_{k}\right)=G\left(\omega_{k}\right)+F^{-1}\left(\omega_{k}\right) .
$$

For large signal-to-noise ratios one can use the approximation $\left(\mathbf{R}\left(\omega_{k}\right)-\mathbf{V}\left(\omega_{k}\right)\right)^{-1} \approx \mathbf{R}^{-1}\left(\omega_{k}\right)$, which gives

$$
\tilde{G}\left(\omega_{k}\right) \approx \mathbf{V}\left(\omega_{k}\right) \mathbf{R}^{-1}\left(\omega_{k}\right)\left(G\left(\omega_{k}\right)+F^{-1}\left(\omega_{k}\right)\right) .
$$

An interesting result regarding the controller is that a high gain controller will give a smaller estimation error. This could at first seem to be in conflict with standard results for spectral analysis (see e.g. Problem 6G.1 in [1]), claiming that $F$ should be as small as possible. The difference is explained by the fact that in [1], the controller $u(t)=-F(q) y(t)+r(t)$ is used, compared to $u(t)=$ $F(q)(r(t-y(t))$ in our setup.

Consider now the case described in (6), Section III, that the same scalar reference signal $r_{0}(t)$ is used in all experiments and for all channels using the permutation matrix $T$. Equation (20) then gives

$$
\tilde{G}\left(\omega_{k}\right) \approx \frac{1}{R_{0}\left(\omega_{k}\right)} \mathbf{V}\left(\omega_{k}\right) T\left(G\left(\omega_{k}\right)+F^{-1}\left(\omega_{k}\right)\right) .
$$

Using this expression, 3 properties of the relative error will be described:

- Non-symmetric relative error,

- Larger relative error for small elements (given that the disturbances $\mathbf{V}_{i j}$ have similar character),

- Relative error dependent on $T$.

\section{A. Non-symmetric Relative Error}

For easier notation, consider the $T_{1}$-case and a diagonal controller. Equation (21) then implies the expressions

$$
\begin{aligned}
& \tilde{G}_{1,3}=\frac{1}{R_{0}}\left(\mathbf{V}_{1,:} G_{:, 3}+\mathbf{V}_{1,3} F_{3,3}^{-1}\right) \\
& \tilde{G}_{3,1}=\frac{1}{R_{0}}\left(\mathbf{V}_{3,:} G_{:, 1}+\mathbf{V}_{3,1} F_{1,1}^{-1}\right)
\end{aligned}
$$

where $\mathbf{V}_{1, \text { : }}$ is row 1 in $\mathbf{V}, G_{:, 3}$ is column 3 in $G$, etc. (argument $\left(\omega_{k}\right)$ omitted for easier notation). In these equations $\mathbf{V}_{i, j}\left(\omega_{k}\right)$ denotes the DFT of the disturbance acting on channel $i$ in experiment $j$.

Equations (22)-(23) explain why the relative error will be non-symmetric. The error $\tilde{G}_{3,1}$ is influenced by the elements in the first column of $G$. The error $G_{1,3}$ is on the other hand influenced by the elements in the third column of $G$, and this will obviously not give the same result.

\section{B. Larger Relative Error for Small Elements}

The relative error will in general be larger for small elements in $G$, which can be seen as follows. By comparing the expression

$$
\tilde{G}_{1,1}=\frac{1}{R_{0}}\left(\mathbf{V}_{1,:} G_{:, 1}+\mathbf{V}_{1,1} F_{1,1}^{-1}\right)
$$

with $\tilde{G}_{3,1}$ from (23) and using the assumption that all $\mathbf{V}_{i j}$ have same character, the magnitude of $\tilde{G}_{1,1}$ and $\tilde{G}_{3,1}$ will be approximately the same. Dividing by $G_{1,1}$ and $G_{3,1}$, respectively, then gives that the relative error will be larger in magnitude for the smaller element, typically for the offdiagonal element.

\section{Dependence on $T$}

If the $\mathbf{V}_{i j}$ are independent of the excitation, the $T_{2}$ case will give lower relative error than the $T_{1}$ case. This is due to the fact that $\mathbf{V R} \mathbf{R}^{-1}=1 / R_{0} \mathbf{V} T^{-1}$ for the $T_{2}$ case involves averaging two elements in $\mathbf{V}$, which obviously will reduce the variance. In particular, if the $\mathbf{V}_{i j}$ are uncorrelated, the variance will be reduced by a factor 2 .

For the robot application, $\mathbf{V}_{i j}$ depends on the movement of the robot, and hence also on the excitation (see Section VA for details). This will affect the assumption of same magnitude for all $\mathbf{V}_{i j}$ and in addition make the components in $\mathbf{V}$ correlated. The choice of permutation matrix $T$ is therefore much more involved for the robot application. 


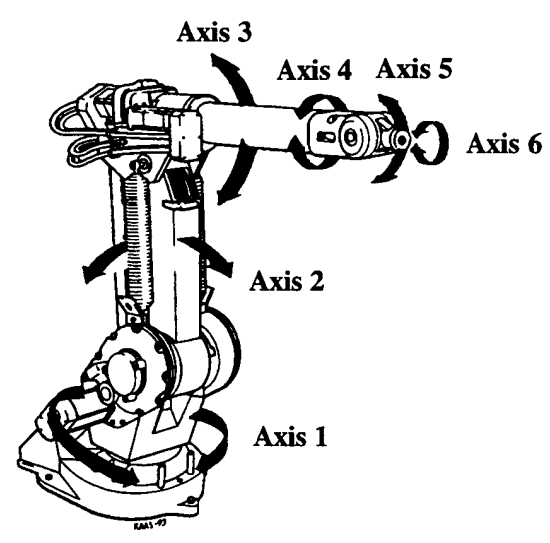

Fig. 2. The ABB manipulator IRB 1400.
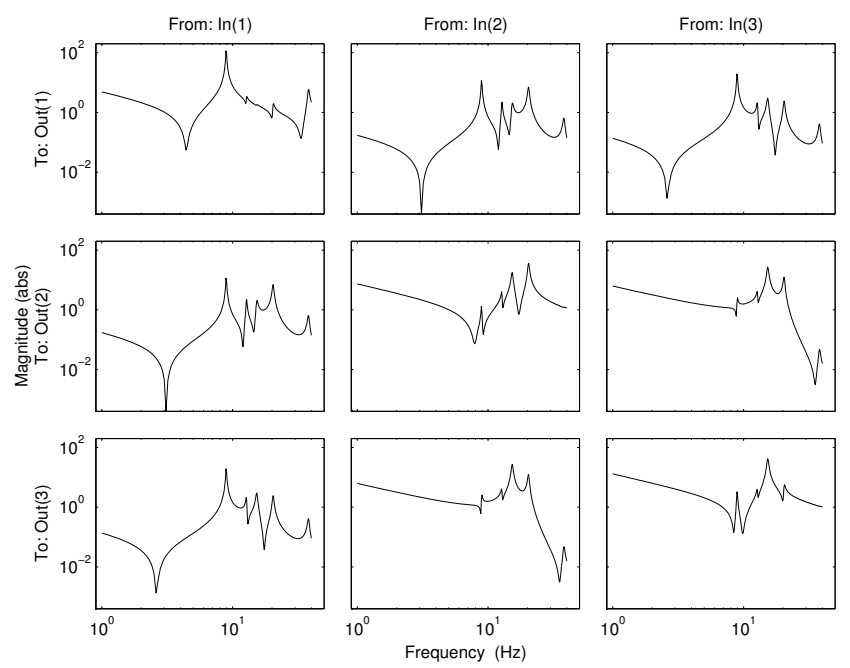

Fig. 3. Bode diagram of the transfer function from motor torque $\tau$ to motor speed $\dot{\varphi}$.

\section{NUMERICAL EXAMPLE}

\section{A. System description}

The robot simulation model used in this paper corresponds to an experimental robot with a load capacity of $250 \mathrm{~kg}$. The model is a linearized version of a non-linear state-space model with 20 states, describing the dynamics of axes 1, 2 and 3 (see Fig. 2) from applied motor torque $\tau$ to achieved motor position $\varphi$.

The non-linear robot model is linearized at zero position and velocity, corresponding to the position in Fig. 2, and a Bode diagram from motor torque $\tau$ to motor speed $\dot{\varphi}$ can be seen in Fig. 3.

As mentioned earlier, the data collection will be carried out in closed loop, which is schematically depicted in Fig. 4. For this kind of application it is necessary to use feedback control while data are collected, in order to keep the robot around its operation point. Limitations according to real life experiments are imposed on the experiments, such as limitations in amplitude and bandwidth for the motor

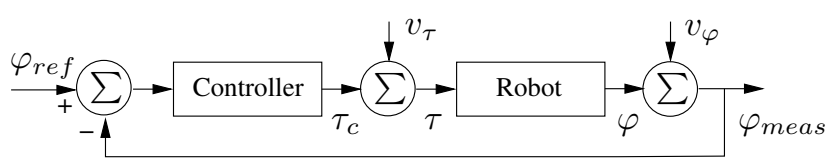

Fig. 4. Block diagram.

TABLE I

NUMERICAL VALUES FOR $v_{\varphi}(t)$ IN (25).

\begin{tabular}{c|c|c}
\hline$n$ & $c_{n}(\mathrm{mrad})$ & $\phi_{c, n}(\mathrm{rad})$ \\
\hline 2 & 0.40 & -1.84 \\
10 & 0.20 & 0.81 \\
12 & 0.22 & 2.94 \\
48 & 0.18 & 2.94 \\
\hline
\end{tabular}

torque, speed, and acceleration.

Measurements $\varphi_{\text {meas }}$ of the motor position $\varphi$ are normally obtained by using Tracking Resolver-to-Digital Converters [8]. It is shown in [8] that the position error $v_{\varphi}$, due to non-ideal resolver characteristics, can be be described as a sum of sinusoids like in

$$
v_{\varphi}(t)=\sum_{n \in \mathbb{N}_{c}} c_{n} \sin \left(n \varphi(t)+\phi_{c, n}\right),
$$

with amplitudes $c_{n}$, phases $\phi_{c, n}$, and $\mathbb{N}_{c}$ a set of integers. Numerical values which are considered to be relevant for the robot application (see e.g. [9]) can be seen in Table I. The signal $v_{\varphi}(t)$ is hereafter denoted (periodic) output disturbance.

Industrial robots usually have AC permanent magnet motors as actuators, which will give rise to ripple in the torque. The ripple can be modeled as sums of sinusoids similar to (25) but with additional terms proportional to the motor current, see [10] for details. However, in this article, only output disturbance is considered.

Some simplifying conditions in the system setup are that the simulation model does not include non-linearities such as, e.g., backlash in the gear-box, static friction, and nonlinear stiffness in the springs. These non-linearities would affect the estimation quality, which has been studied in ,e.g., [11] for black-box identification of an industrial robot, and would be interesting to incorporate in a future study.

\section{B. Identification experiment}

The motor speed $\dot{\varphi}(t)$ will be used as output $y(t)$ in the identification, and the excitation signal $\mathbf{r}(t)$ is therefore applied as reference speed for the controller, which can be seen as a PI-controller for speed. Hence, $\varphi_{\text {ref }}(t)$ in Fig. 4 is the integral of the reference signal $r(t)$.

The controller $F$ used in the experiments is diagonal, and the diagonal elements are of PI-type. The high frequency gains of their inverses tend to 0.4 as $\omega$ tends to infinity. The cut-off frequencies of the inverses are around $1 \mathrm{rad} / \mathrm{s}$.

The reference speed signal has a peak value of $8 \mathrm{rad} / \mathrm{s}$, signal period $T_{0}=10 \mathrm{~s}$, waiting time $T_{w}=20 \mathrm{~s}$, and its frequency contents in the interval $[1,40] \mathrm{Hz}$. Using 

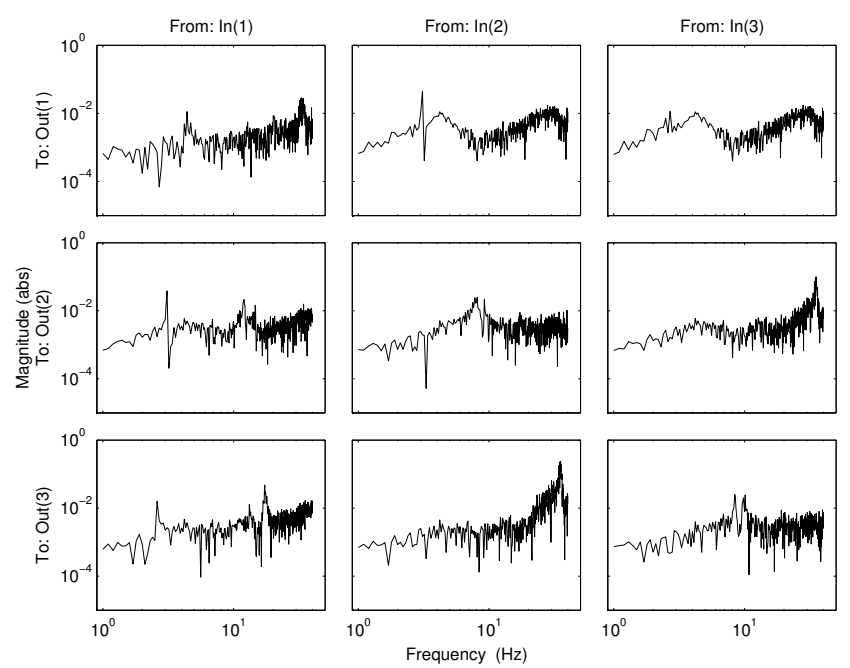

Fig. 5. $|\Delta G|$ for $T=T_{1}$ and periodic disturbances.

TABLE II

$\overline{|\Delta G|}($ IN \%) FOR PERIODIC DISTURBANCES.

\begin{tabular}{ccc|ccc}
\hline \multicolumn{3}{c|}{$T=T_{1}$} & \multicolumn{3}{c}{$T=T_{2}$} \\
\hline 0.40 & 0.58 & 0.57 & 0.40 & 0.87 & 0.61 \\
0.43 & 0.39 & 0.81 & 1.99 & 0.25 & 6.84 \\
0.55 & 1.65 & 0.35 & 1.41 & 8.93 & 0.24 \\
\hline
\end{tabular}

the multisine signal (8) with random initial phases and crest factor optimization with 100 iterations, gives a rootmean-square (RMS) value of $4.9 \mathrm{rad} / \mathrm{s}$. The resulting signal amplitudes correspond to realistic real life experiments on a robot with comparable size.

Since the motor speed $\dot{\varphi}(t)$ is used as output, the corresponding output disturbance, denoted $v_{y}(t)$, will therefore be the derivative $\dot{v}_{\varphi}(t)$ of the periodic disturbance $v_{\varphi}(t)$ in (25). The RMS value for the matrix $\mathbf{v}_{\mathbf{y}}(t) \in \mathbb{C}^{p \times m}$ will depend on the particular excitation signal $\mathbf{r}(t)$. For the $T_{2}$ case, the RMS values for the elements in $\mathbf{v}_{\mathbf{y}}(t)$ are all approximately $0.03 \mathrm{rad} / \mathrm{s}$. For the $T_{1}$ case, the off-diagonal elements are approximately 10 times lower.

For comparison, stochastic (Gaussian) disturbances will be used as well. These are chosen to get the same power and frequency contents as in the $T_{2}$ case for period disturbances. For the $T_{1}$ case, one should therefore not compare the results for periodic and stochastic disturbances.

The absolute relative error $|\Delta G|$ for periodic disturbances can be seen in Fig. 5 for $T=T_{1}$ and Fig. 6 for $T=T_{2}$. See also Table II for $\overline{|\Delta G|}$.

For stochastic disturbances, 500 Monte Carlo simulations have been made. Then the mean and standard deviation of $\overline{|\Delta G|}$ and $|\Delta G|$ are calculated with respect to (w.r.t.) realization. The (averaged) absolute relative error $|\Delta G|$ can be seen in Fig. 7. See also Table III for $\overline{|\Delta G|}$ (average \pm one standard deviation).

Remark 1: Note the difference if the order of taking absolute value and taking mean and standard deviation w.r.t.

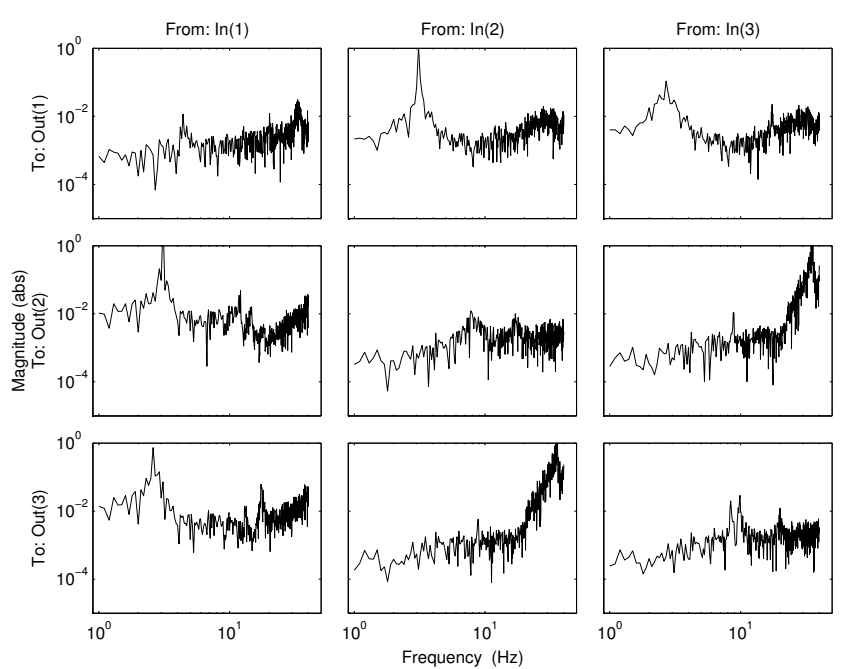

Fig. 6. $|\Delta G|$ for $T=T_{2}$ and periodic disturbances.

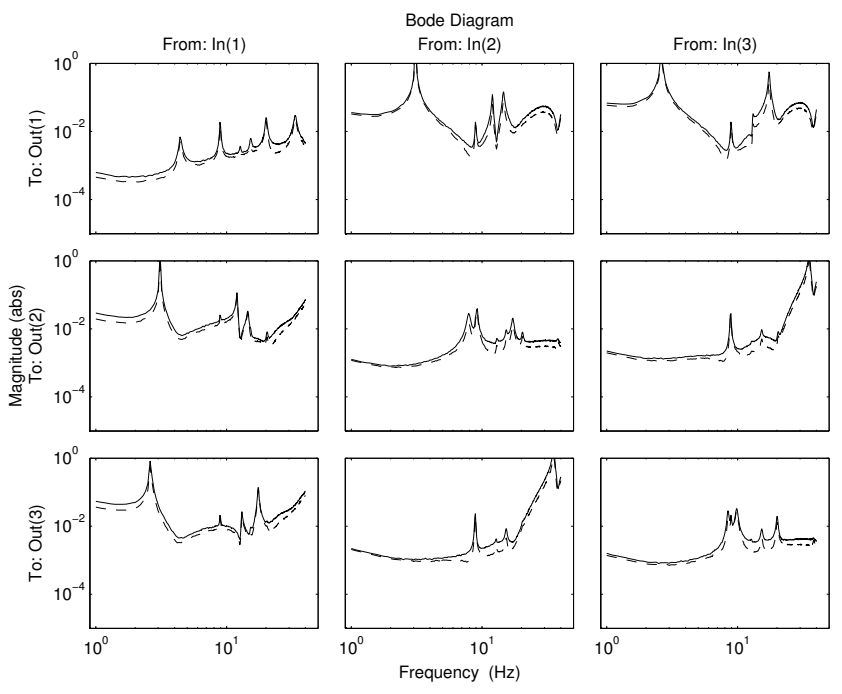

Fig. 7. $|\Delta G|$ with stochastic disturbances (averaged over 500 realizations) for $T=T_{1}$ (solid line) and $T=T_{2}$ (dashed line).

realization is switched. Averaging the complex values would give much lower values and is similar to the averaging technique mentioned in Section II. However, that is not the issue here, we are mainly interested in knowing how a single estimate varies depending on the realization and therefore the absolute values are averaged.

Remark 2: Monte Carlo simulations w.r.t. the excitation signal would also be interesting to carry out, especially since the properties of the periodic disturbance depends on the excitation signal. Preliminary work hints that averaging over experiments with slightly varying operating points will reduce the relative error in the estimations.

\section{Experimental results}

From the results presented in Fig. 5-7 and Tables II-III a number of observations can be made: 
TABLE III

$\overline{|\Delta G|}$ (AVERAGE \pm ONE STANDARD DEVIATION, IN \%) FOR STOCHASTIC DISTURBANCES.

\begin{tabular}{c|ccc}
\hline \multirow{3}{*}{$T=T_{1}$} & $0.62 \pm 0.03$ & $4.64 \pm 0.65$ & $5.84 \pm 0.43$ \\
& $2.52 \pm 0.25$ & $0.56 \pm 0.02$ & $13.32 \pm 0.81$ \\
& $3.32 \pm 0.32$ & $16.87 \pm 1.01$ & $0.50 \pm 0.02$ \\
\hline \multirow{3}{*}{$T=T_{2}$} & $0.46 \pm 0.02$ & $3.45 \pm 0.53$ & $4.01 \pm 0.34$ \\
& $1.85 \pm 0.18$ & $0.37 \pm 0.02$ & $9.17 \pm 0.58$ \\
& $2.44 \pm 0.21$ & $11.33 \pm 0.65$ & $0.34 \pm 0.02$ \\
\hline
\end{tabular}

- Even though the multivariable system itself, shown in Fig. 3, is symmetric the relative error is nonsymmetric. This is the case for both periodic and stochastic disturbances.

- In all cases presented above the relative error of the offdiagonal elements is in general larger than the relative error of the diagonal elements.

- For the stochastic disturbance $T=T_{2}$ gives smaller relative error for all elements.

- For the periodic disturbance the choice $T=T_{2}$ gives smaller relative error for the diagonal elements in $\hat{G}\left(\omega_{k}\right)$ but higher relative error for the off-diagonal elements.

The first three observations are explained by the error analysis in Section IV. For the last observation, some additional analysis is needed.

For the periodic disturbance case the situation for $T_{2}$ is the opposite, compared to the stochastic case. The main reason is that the stochastic disturbance is assumed to be additive while the properties of the periodic disturbance depend on the movement of the robot, and hence also on the excitation. Since the measured motor speed $\dot{\varphi}_{\text {meas }}(t)$ is considered as output $y(t)$, the disturbance $v_{y}(t)$ is $\dot{v}_{\varphi}(t)$ like

$$
v_{y}(t)=\dot{v}_{\varphi}(t)=\dot{\varphi}(t) \sum_{n \in \mathbb{N}_{c}} c_{n} n \cos \left(n \varphi(t)+\phi_{c, n}\right)
$$

which hints that $v_{y}(t)$ is highly correlated with $\dot{\varphi}(t)$. Due to the controller, we have, in the sample points, the relation $\dot{\varphi}(t)=G_{c}(q) r(t)$, where $G_{c}(q)$ is the closed loop system and $q$ is the shift operator. We therefore get the approximation

$$
\mathbf{V}_{y}\left(\omega_{k}\right) \approx \text { const } \cdot G_{c}\left(\omega_{k}\right) \mathbf{R}\left(\omega_{k}\right)
$$

which gives useful understanding and seems to be fairly accurate for the excited frequencies in the present simulations. To be noted is that the nonlinear relation (26) introduces leakage to neighboring frequencies and therefore $V_{y}\left(\omega_{k}\right) \neq 0$ even though $R\left(\omega_{k}\right)=0$.

Using $\mathbf{R}\left(\omega_{k}\right)=T R_{0}\left(\omega_{k}\right)$ then gives

$$
\mathbf{V}_{y}\left(\omega_{k}\right) \approx \text { const } \cdot G_{c}\left(\omega_{k}\right) T R_{0}\left(\omega_{k}\right)
$$

which shows that the disturbances will be drastically different in the $T_{1}$ and $T_{2}$ cases. Using $G_{c} \approx I$ gives that in the $T_{1}$ case, $\mathbf{V}_{y}$ will be approximately diagonal, whereas in the $T_{2}$ case, all elements in $\mathbf{V}_{y}$ will have approximately the same size. This partly explains the RMS values mentioned in Section V-B.

Using the approximation (28) in (20) with $F^{-1}+G=$ $G_{c}^{-1} G$ then gives that the relative error $\Delta G \approx$ const, independent of $T$. This is, from Fig. 5-6, obviously not the case, but at least $|\Delta G|$ varies less for the periodic case compared to the stochastic case. To find the explanation to the major differences in the $T_{1}$ and $T_{2}$ cases, a better approximation for $\mathbf{V}_{y}$ is needed.

\section{CONCLUSIONS}

A method for estimating the Multivariable Frequency Response Function (MFRF) for closed loop data has been studied. An approximate expression for the estimation error has been derived, and using this expression some properties of the estimation error have been pointed out. In particular, the expression describe how the MFRF estimate is affected by the properties of the disturbances, the choice of excitation signal in the different input channels, the feedback and the properties of the system itself. As a numerical example, a linearized model of an industrial robot has been used, showing that the approximate error expression can explain some peculiar results such as non-symmetric relative error.

\section{ACKNOWLEDGMENTS}

This work was supported by VINNOVA's Center of Excellence ISIS at Linköpings universitet.

\section{REFERENCES}

[1] L. Ljung, System Identification: Theory for the User, 2nd ed. Upper Saddle River, New Jersey, USA: Prentice Hall, 1999.

[2] R. Pintelon and J. Schoukens, System identification: a frequency domain approach. New York: IEEE Press, 2001.

[3] P. Guillaume, R. Pintelon, and J. Schoukens, "Accurate estimation of multivariable frequency response functions," in Proceedings of the 13th IFAC Triennial World Congress, San Francisco, 1996, pp. 423-428.

[4] K. Kozlowski, Modelling and identification in robotics, ser. Advances in Industrial Control. London: Springer, 1998.

[5] R. Pintelon and J. Schoukens, "Measurement of frequency response functions using periodic excitations, corrupted by correlated input/output errors," IEEE Trans. Instrum. Meas., vol. 50, no. 6, pp. 1753-1760, Dec. 2001.

[6] J. Schoukens, R. M. Pintelon, and Y. J. Rolain, "Broadband versus stepped sine FRF measurements," IEEE Trans. Instrum. Meas., vol. 49 , no. 2 , pp. $275-278$, Apr. 2000.

[7] E. V. der Ouderaa, J. Schoukens, and J. Renneboog, "Peak factor minimization using a time-frequency domain swapping algorithm," IEEE Trans. Instrum. Meas., vol. 37, no. 1, pp. 145-147, Mar. 1988.

[8] D. Hanselman, "Resolver signal requirements for high accuracy resolver-to-digital conversion," IEEE Trans. Ind. Electron., vol. 37, no. 6, pp. 556-561, Dec. 1990.

[9] E. Uddeholt, Identifiering och kompensering av motor- och resolverrippel, Master thesis IR-RT-EX-9806, Dept. of Signals, Sensors \& Systems, Royal Institute of Technology, SE-100 44 Stockholm, Sweden, 1998.

[10] H.-J. Gutt, F. Scholl, and J. Blattner, "High precision servo drives with DSP-based torque ripple reduction," in Proceedings of IEEE AFRICON, 1996, vol. 2, Sep. 1996, pp. 632-637.

[11] M. Aberger, "Effects of nonlinearities in black box identification of an industrial robot," Department of Electrical Engineering, Linköping University, SE-581 83 Linköping, Sweden, Tech. Rep. LiTH-ISY-R2322, Dec 2000. 